\title{
Perception du bien-être animal par les étudiants de I'Université Cheikh Anta Diop de Dakar
}

\author{
Marie Fausta Dutuze ${ }^{1 *}$ Abdoul Razak Issa Garba ${ }^{2}$ \\ Rosine Manishimwe ${ }^{1}$ Philippe Kone ${ }^{3}$
}

\section{Mots-clés}

Bien-être animal, douleur, sensibilisation, étudiant, productivité, Sénégal

Accepted: 20 January 2016; Published: 9 May 2016

\section{Résumé}

Le bien-être animal (BEA) est un concept très complexe à définir mais important pour la bonne qualité des produits d'élevage. Afin d'évaluer la perception de ce concept, une étude a été menée au sein de la communauté estudiantine de I'Université Cheikh Anta Diop de Dakar (UCAD) entre le 16 janvier et le 2 mai 2012. Pendant cette période la population estudiantine était de 84396. En tout 168 étudiants ont été interviewés à I'aide d'un questionnaire structuré. L'étude a montré que seulement $57 \%$ d'entre eux avaient entendu parler au moins une fois du BEA, dont 60,4\% à travers les médias. Les connaissances des étudiants sur le BEA ont varié d'une faculté à l'autre : les étudiants de la Faculté des sciences et technologie de l'éducation et de la formation (Fastef) étaient les mieux renseignés. Parmi nos enquêtés, 73,2 \% pensaient que les animaux ressentent la douleur et $93 \%$ qu'il existe une relation entre le BEA et la productivité. Une sensibilisation sur ce concept est nécessaire et doit se faire à travers les médias qui constituent le canal le plus utilisé par les étudiants de l'UCAD.

- Comment citer cet article : Dutuze M.F., Issa Garba A.R., Manishimwe R., Kone P., 2015. Perception of animal welfare by Cheikh Anta Diop University's students [in French]. Rev. Elev. Med. Vet. Pays Trop., 68 (4): $151-156$

\section{INTRODUCTION}

Le bien-être animal (BEA) est aujourd'hui au cœur de nombreuses discussions et débats philosophiques, sociaux, économiques, scientifiques et politiques. On entend par bien-être animal la manière dont un animal évolue dans les conditions qui l'entourent (OMS, 2010). Le Farm Animal Welfare Council (1992) définit le BEA à travers les cinq libertés indispensables au bien-être des animaux :

\footnotetext{
1. School of Animal Sciences and Veterinary Medicine, College of Agriculture, Animal Sciences and Veterinary Medicine, University of Rwanda, BP 57. Nyagatare, Rwanda.

2. Ministère de l'Elevage / Université Dan Dicko Dankoulodo, Maradi, Niger. 3. Service de microbiologie / immunologie / pathologie infectieuse, Département santé publique et environnement, Ecole inter-Etats des sciences et médecine vétérinaires de Dakar, Dakar-Fann, Sénégal.

* Auteur pour la correspondance

Tél. : +250787363943; +250722246962

Email :dkmfausta6@yahoo.fr
}

l'absence de faim, de soif ou de malnutrition ; la présence d'abris appropriés et le confort; l'absence de maladie et de blessures ; l'absence de peur et d'anxiété ; et la possibilité d'exprimer les comportements normaux de l'espèce ; ces cinq libertés ont toutes un poids identique pour le BEA. Le BEA requiert les éléments suivants : prévention et traitement des maladies, protection appropriée, soins, alimentation adaptée, manipulations réalisées sans cruauté, abattage ou mise à mort effectués dans des conditions décentes (OMS, 2010).

En 2000, l'Organisation mondiale de la santé animale (OIE) a défini le BEA comme domaine d'action prioritaire dans le cadre de son plan stratégique couvrant la période 2001-2005. Dans le cadre du cinquième plan stratégique (2011-2015), l'OIE a poursuivi ses travaux sur le bien-être des animaux utilisés pour la recherche et l'enseignement, et dans les systèmes de production animale, dont les poissons d'élevage. Pour cela l'OIE a mis en place des règles et des normes internationales sur le BEA basées sur des résultats scientifiques. Trois conférences internationales ont été organisées : à Paris en 2004, au Caire en 2008 et en Malaisie en 2012 (OMS, 2012). 
Diverses études et enquêtes d'opinion, d'évaluation des connaissances et de la perception du BEA ont été menées dans différents pays (Australie, Etats-Unis, Nouvelle-Zélande, France, Belgique, Grande-Bretagne, entre autres) (Coleman, 2009 ; Heleski et Zanella, 2006 ; Williams et al., 2007). Cependant, très peu d'études ont été réalisées en Afrique jusqu'à présent. Pour donner une idée de la perception du BEA en Afrique, cette étude a été réalisée au sein de l'Université Cheikh Anta Diop de Dakar (ou Université de Dakar, ou UCAD). L'UCAD est la principale université du Sénégal. Elle est la plus ancienne université d'Afrique noire d'expression française et fait partie des plus renommées sur le continent. Elle forme les cadres d'Afrique de l'Ouest et de l'Afrique en général. Cette étude a eu pour objectif de donner une idée générale de la conception du BEA chez les futurs cadres africains, surtout d'Afrique de l'Ouest.

\section{MATERIEL ET METHODES}

Les étudiants de l'université Cheikh Anta Diop sont originaires de nombreux pays d'Afrique francophone : Afrique de l'Ouest, Maghreb, Gabon, Cameroun, République centrafricaine, République démocratique du Congo, entre autres. Des pays francophones d'autres continents sont également représentés à l'UCAD (par exemple France, Belgique, Liban, Canada). Les étudiants poursuivent des formations dans des domaines variés, notamment l'éducation, la santé, la médecine, les sciences humaines, l'économie, la gestion, les sciences juridiques, les sciences politiques, les sciences sociales, la technologie. Toutes les classes d'âge sont représentées. L'UCAD acceuillait 84396 étudiants pendant la période d'étude du 16 janvier au 2 mai 2012.

Cette université comprend six facultés, trois écoles, un centre de formation, un institut de formation et douze instituts d'université (UCAD, 2012). L'étude a porté sur les six facultés, deux écoles, un centre de formation et un institut de formation : la Faculté des sciences et techniques (FST), la Faculté de médecine, pharmacie et odonto-stomatologie (Fmpos), la Faculté des lettres et sciences humaines (FLSH), la Faculté des sciences économiques et de gestion (Faseg), la Faculté des sciences et technologies de l'éducation et de la formation (Fastef), la Faculté des sciences juridiques et politiques (FSJP), l'Ecole supérieure polytechnique (ESP), l'Ecole des bibliothécaires, archivistes et documentalistes (EBAD), le Centre d'études des sciences et techniques de l'information (Cesti) et l'Institut national supérieur de l'éducation et du sport (Inseps). Parmi eux, l'Ecole inter-Etats des sciences et médecine vétérinaires (Eismv) a été exclue car une étude similaire y avait été conduite l'année précédente (Adjé Koffi, 2011).

La taille de l'échantillon était de 168. Elle a été calculée avec le logiciel Win Episcope 2.0 en considérant le niveau des connaissances sur le BEA des acteurs de l'Eismv de Dakar qui est de 87,7 \% (Adjé Koffi, 2011) avec un niveau de confiance de $95 \%$. Le nombre de personnes à interroger dans chacune des entités a été calculé par échantillonnage stratifié proportionnel.

Un questionnaire structuré ( $c f$. matériel supplémentaire) a été utilisé pour récolter les données. Les fiches d'enquête ont été remplies par les enquêtés eux-mêmes en présence et avec l'aide des enquêteurs. Les données ont été saisies avec le logiciel EpiData 3.1, puis traitées et visualisées avec Excel 2007. L'analyse statistique a été réalisée avec le logiciel R 2.13.0. Les tests du $\mathrm{Chi}^{2}$ et l'Anova ont été les principaux tests utilisés. Le niveau de significativité a été fixé à $\mathrm{p}<0,05$. Une échelle de Likert a été utilisée pour classer les opinions. C'est une échelle de jugement, avec laquelle la personne interrogée exprime son degré d'accord ou de désaccord vis-à-vis d'une affirmation. L'échelle contient en général trois à sept choix de réponses qui permettent de nuancer le degré d'accord. Une note est attribuée à chaque réponse proposée, ce qui permet un traitement quantitatif des données. Le texte des étiquettes est variable (Altinok, 2005). Dans notre étude, trois types d'échelles ont été utilisés : $1=$ pas du tout, $2=$ un peu, $3=$ plus ou moins, 4 = beaucoup ; 1 = en total désaccord, $2=$ plus ou moins pas d'accord, $3=$ neutre, $4=$ plus ou moins en accord, $5=$ tout à fait d'accord ; et 1 = très défavorable, $2=$ moyennement défavorable, $3=$ un peu défavorable, $4=$ neutre, $5=$ un peu favorable, $6=$ moyennement favorable, $7=$ très favorable.

\section{RESULTATS}

Parmi les 168 étudiants interrogés, 119 (71\%) étaient de sexe masculin et $49(29 \%)$ de sexe féminin. Le tableau I montre la répartition des étudiants par classe d'âge, faculté ou école, et année d'étude.

\section{Relation avec les animaux}

Sur les 168 étudiants, $45 \%$ possédaient des animaux. Parmi eux, $70 \%$ avaient des animaux d'élevage, $18 \%$ des animaux de compagnie et seulement $12 \%$ les deux types d'animaux. Plus d'un tiers $(37,1 \%)$ aimaient beaucoup les animaux. L'échelle de Likert a montré qu'en général les étudiants de l'UCAD aimaient plus ou moins les animaux avec un score moyen de 3,00 $\pm 0,14$.

\section{Connaissances générales sur le BEA}

Plus de la moitié (57\%) des étudiants avaient entendu parler au moins une fois de la notion de BEA. Les avis étaient significativement liés à la possession d'animaux $(\mathrm{p}=0,008922)$, à la faculté, école ou institut $\left(\mathrm{p}<2,2 \cdot 10^{-16}\right)$. Les étudiants qui possédaient des animaux et ceux de la Fastef étaient davantage renseignés sur la notion du BEA, dans des proportions respectives de 71 et $67,1 \%$. Parmi les étudiants $60,4 \%$ avaient entendu parler de la notion de BEA à travers les médias et seulement 9,3\% à travers les cours à l'université.

Seulement $4,8 \%$ des enquêtés ont donné la définition complète du BEA constituée des cinq libertés et $24 \%$ connaissaient au moins trois des cinq principes. La troisième liberté (bonne santé et absence de blessure et/ou de douleurs) était la plus connue $(73,9 \%)$, tandis que la cinquième (absence de peur et de détresse) n'était connue que de $16 \%$ des enquêtés.

\section{Respect du bien-être animal dans les conditions d'élevage africaines}

Parmi les étudiants 67,6\% étaient d'accord avec le fait que le BEA est une question primordiale en Afrique. Cependant, l'échelle de Likert a montré qu'ils étaient plus ou moins d'accord avec un score moyen de 3,78 $\pm 0,2$. De plus, il existait une différence significative par rapport à l'âge $(\mathrm{p}=0,03902)$ : les étudiants âgés entre 15 et 26 ans étaient plus en accord avec le fait que le BEA est une question primordiale en Afrique (80\%). En général, environ un tiers $(32,1 \%)$ des étudiants étaient plus ou moins en accord avec le fait que le BEA est respecté dans les conditions d'élevage en Afrique.

\section{Sensibilité des animaux et utilisation des animaux dans la recherche scientifique}

Parmi les interviewés 73,2 \% pensaient que les animaux ressentent la douleur comme les humains. Concernant l'utilisation des animaux dans la recherche scientifique, $64 \%$ y étaient favorables, $20 \%$ défavorables et $16 \%$ neutres. L'échelle de Likert a montré que de manière générale ils y étaient un peu favorables avec un 
score moyen de 4,94 $\pm 0,28$. Il existe une différence significative entre les différentes facultés, écoles, centre et institut ( $p<2,2 \mathrm{e}-16)$. En effet les étudiants de Fastef et Fmpos étaient très favorables à l'utilisation des animaux dans la recherche scientifique (scores respectifs de 5,72 $\pm 1,94$ et de $5,63 \pm 2,01$ ).

\section{Bien-être animal et exploitation des animaux}

Parmi les étudiants $92,8 \%$ considéraient qu'il y avait une relation entre le BEA et la productivité, et $89,8 \%$ pensaient que la prise en compte du BEA dans les conditions d'élevage en Afrique permettrait d'augmenter la productivité. Leur réponse a été en général neutre sur la question de l'utilisation des animaux en exploitation agricole (score moyen de 4,25 $\pm 0,32$ ). Par ailleurs, $59 \%$ pensaient que de manière générale les animaux étaient maltraités, $17 \%$ pensaient le contraire, et $24 \%$ affirmaient ignorer les conditions des animaux exploités dans le secteur agricole. Près de $40 \%$ des étudiants affirmaient que le concept du BEA n'était pas respecté dans le domaine du transport, 48,8 \% pensaient le contraire et $11,9 \%$ se sont abstenus. Ces avis ont été significativement influencés par le sexe $(p=0,0205)$ et le niveau d'étude $(p=0,03419)$. Les étudiants de première année et les étudiantes en général ont été plus nombreux à penser que le BEA n'était pas respecté dans le domaine du transport (respectivement 57,4 et 69,9\%).

\section{Tableau I}

Résultats principaux de l'enquête sur la perception du bien-être animal (BEA) par les étudiants de I'université Cheikh Anta Diop de Dakar au Sénégal

\begin{tabular}{|c|c|c|c|c|c|c|c|}
\hline & & $\begin{array}{l}\text { Effectif } \\
(n=168)\end{array}$ & Ont entendu ${ }^{1}$ & Connaissent $^{2}$ & $\begin{array}{l}\text { Utilisation dans } \\
\text { la recherche } \\
\end{array}$ & $\begin{array}{c}\text { Question } \\
\text { primordiale } \\
\text { en Afrique }\end{array}$ & $\begin{array}{c}\text { BEA et } \\
\text { productivité } 5\end{array}$ \\
\hline \multicolumn{2}{|l|}{$\begin{array}{l}\text { Echantillon } \\
(\%)\end{array}$} & & $98(57)$ & $40(24)$ & $\begin{array}{l}\text { Score moyen } \\
4,9 \pm 0,3\end{array}$ & $\begin{array}{l}\text { Score moyen } \\
3,8 \pm 0,2\end{array}$ & $\begin{array}{l}\text { Sont en accord }(\%) \\
\quad 155(92,8)\end{array}$ \\
\hline \multirow{3}{*}{$\begin{array}{l}\text { Sexe } \\
(\%)\end{array}$} & Masculin & 119 & $67(53,7)$ & $28(23,5)$ & $4,9 \pm 1,9$ & $3,8 \pm 1,4$ & $110(92,4)$ \\
\hline & Féminin & 49 & $32(65,3)$ & $13(26,5)$ & $4,8 \pm 1,9$ & $3,8 \pm 1,4$ & $46(93,8)$ \\
\hline & Significativité & & NS & NS & NS & NS & NS \\
\hline \multirow{5}{*}{$\begin{array}{l}\text { Age } \\
(\%)\end{array}$} & [15-20 ans] & 12 & $7(58,3)$ & $1(8,3)$ & $4,9 \pm 1,9$ & $3,7 \pm 1,4$ & $10(83,3)$ \\
\hline & [21-26 ans] & 111 & $68(61,2)$ & $26(23,6)$ & $4,9 \pm 1,9$ & $3,8 \pm 1,4$ & $101(91,8)$ \\
\hline & [27-32 ans] & 38 & $17(44,7)$ & $10(26,3)$ & $4,8 \pm 1,9$ & $3,8 \pm 1,4$ & $37(97,3)$ \\
\hline & $>32$ ans & 7 & $4(57,1)$ & $3(42,8)$ & $4,9 \pm 1,9$ & $3,8 \pm 1,4$ & 7 (100) \\
\hline & Significativité & & NS & $p=0,022$ & NS & $p=0,039$ & NS \\
\hline \multirow{11}{*}{$\begin{array}{l}\text { Facultés et } \\
\text { écoles au sein } \\
\text { de l'université } \\
(\%)\end{array}$} & Faseg & $14(8,3)$ & $8(57,1)$ & 14,2 & $4,5 \pm 1,7$ & $4,1 \pm 1,4$ & $14(100)$ \\
\hline & Fastef & $10(5,9)$ & $8(80)$ & $3(30)$ & $5,7 \pm 1,9$ & $3,5 \pm 1,6$ & $8(80)$ \\
\hline & FLSH & $52(30,9)$ & $26(50)$ & $3(5,7)$ & $4,8 \pm 2,0$ & $3,8 \pm 1,4$ & $50(96,1)$ \\
\hline & Fmpos & $5(3)$ & $2(40)$ & $3(60)$ & $5,6 \pm 2,0$ & $3,0 \pm 1,2$ & $5(100)$ \\
\hline & FSJP & $26(15,5)$ & $16(61,5)$ & $5(19,2)$ & $4,8 \pm 2,1$ & $4,0 \pm 1,3$ & $24(92,3)$ \\
\hline & FST & $49(29,2)$ & $29(59,2)$ & $13(26,5)$ & $4,7 \pm 1,8$ & $3,8 \pm 1,4$ & $45(91,8)$ \\
\hline & Cesti & $3(1,8)$ & $2(66,6)$ & $1(33,3)$ & $4,9 \pm 1,9$ & $3,8 \pm 1,4$ & $3(100)$ \\
\hline & EBAD & $2(1,2)$ & $0(0)$ & $0(0)$ & $4,5 \pm 0,7$ & $4,0 \pm 1,4$ & $2(100)$ \\
\hline & Insep & $4(2,4)$ & $3(75)$ & $1(25)$ & $4,5 \pm 1,4$ & $3,8 \pm 1,4$ & $4(100)$ \\
\hline & ESP & $3(1,7)$ & $2(66,6)$ & $0(0)$ & $4,8 \pm 1,9$ & $3,7 \pm 1,1$ & $1(33,3)$ \\
\hline & Significativité & & $\mathrm{p}<2,2.10^{-16}$ & NS & $p<2,2 e-16$ & NS & NS \\
\hline \multirow{7}{*}{$\begin{array}{l}\text { Niveau d'étude } \\
(\%)\end{array}$} & $1^{\text {re }}$ année & $42(25)$ & $27(64,3)$ & $10(23,8)$ & $4,8 \pm 1,9$ & $3,8 \pm 1,4$ & $37(88,1)$ \\
\hline & $2^{\mathrm{e}}$ année & $32(19)$ & $17(53,1)$ & $7(21,8)$ & $4,8 \pm 1,9$ & $3,8 \pm 1,4$ & $30(93,7)$ \\
\hline & $3^{\mathrm{e}}$ année & $21(12,5)$ & $13(61,9)$ & $0(0)$ & $4,9 \pm 1,9$ & $3,8 \pm 1,4$ & $19(90,5)$ \\
\hline & $4^{\mathrm{e}}$ année & $16(9,5)$ & $8(50)$ & $6(3,7)$ & $4,9 \pm 1,9$ & $3,8 \pm 1,4$ & $15(93,7)$ \\
\hline & $5^{\mathrm{e}}$ année & $41(24,4)$ & $22(53,6)$ & $12(29,2)$ & $4,9 \pm 2$ & $3,8 \pm 1,4$ & $40(97,6)$ \\
\hline & $>5^{\mathrm{e}}$ année & $16(9,5)$ & $9(56,2)$ & $6(3,7)$ & $4,9 \pm 2,1$ & $3,7 \pm 1,4$ & $15(93,7)$ \\
\hline & Significativité & & NS & NS & NS & NS & NS \\
\hline \multicolumn{8}{|c|}{${ }^{1}$ Ont entendu parler de la notion de BEA au moins une fois } \\
\hline \multicolumn{8}{|c|}{2 Connaissent entre trois et cinq principes de liberté des animaux } \\
\hline \multicolumn{8}{|c|}{$\begin{array}{l}3 \text { Opinion sur l'utilisation des animaux dans la recherche scientifique : } 1=\text { très défavorable } ; 2=\text { moyennement défavorable } ; 3=\text { un peu défavorable } ; 4=\text { neutre } ; 5=\text { un peu } \\
\text { favorable } ; 6=\text { moyennement favorable } ; 7=\text { très favorable }\end{array}$} \\
\hline \multicolumn{8}{|c|}{$\begin{array}{l}{ }^{4} \text { Opinion sur le BEA en tant que question primordiale en Afrique : } 1=\text { en total désaccord ; } 2=\text { plus ou moins pas d'accord ; } 3=\text { neutre ; } 4=\text { plus ou moins d'accord } \\
5=\text { tout à fait d'accord }\end{array}$} \\
\hline \multicolumn{8}{|c|}{${ }^{5}$ Considèrent que la prise en compte du BEA permet d'augmenter la productivité } \\
\hline
\end{tabular}




\section{DISCUSSION}

\section{Connaissances générales sur le BEA}

Seulement $57 \%$ des étudiants de l'UCAD ont entendu parler au moins une fois de la notion de BEA. Les medias constituaient la source d'information principale. Une différence significative a été constatée entre les facultés, écoles, centre et institut. Les étudiants de la faculté de Fastef ont davantage entendu parler de la notion de BEA. Ceci peut s'expliquer par le fait qu'ils ont vocation à devenir enseignants et ont donc a priori une plus grande culture générale. A l'Eismv de Dakar 87,7 \% des étudiants, des enseignantschercheurs et du personnel technique et administratif ont entendu parler de la notion de BEA (Adjé Koffi, 2011). Ceci peut s'expliquer par le fait que les acteurs de l'Eismv ont une grande connaissance des sciences animales.

\section{Respect du bien-être animal dans nos conditions d'élevage d'Afrique}

La majorité des enquêtés (63\%) était plus ou moins d'accord avec le fait que le BEA doit être une question primordiale - même si 22,5\% d'entre eux se sont opposés à cet énoncé -, un résultat légèrement inférieur à celui trouvé à l'Eismv, soit $69 \%$ des étudiants, enseignants-chercheurs, et personnel technique et administratif. La raison de cette légère différence pourrait être due au fait que l'étude à l'Eismv impliquait d'autres acteurs que les étudiants, notamment les enseignants-chercheurs qui étaient majoritairement des vétérinaires, alors que notre étude a été réalisée exclusivement au sein d'une communauté estudiantine de disciplines variées.

Une enquête similaire, réalisée en Australie en 2005 auprès de 1061 opérateurs d'élevage, a montré que $60 \%$ des interviewés approuvaient le fait que le bien-être des animaux soit une question fondamentale et $12 \%$ étaient contre (Coleman et al., 2005). En outre, 22,5\% des répondants pensaient que le BEA n'était pas une question primordiale, avec $79,1 \%$ parmi ceux-ci estimant que cette question était certes importante sans être primordiale ; pour eux le BEA ne peut pas être primordial dans les contrées africaines car le bien-être des humains passe avant celui des animaux. Ils affirment que le continent africain est confronté à plusieurs problèmes affectant directement l'espèce humaine qui doivent être résolus en priorité, comme les guerres, les maladies diverses, la famine, l'éducation. Pour cela, selon eux, le BEA n'est pas prioritaire.

Cet avis est contraire à celui de Kant qui dit que « les animaux étant des purs moyens mis à la disposition de l'humanité, la cruauté envers eux constitue une violation du devoir de l'homme envers lui-même » (Burgat, 2002). Ainsi pour Kant, le BEA est une partie intégrante du bien-être des humains alors que selon les réponses obtenues, les humains doivent d'abord s'occuper de leur propre bien-être avant de s'occuper de celui des animaux.

\section{Sensibilité des animaux et utilisation des animaux dans la recherche scientifique}

La majorité des étudiants interviewés $(73,2 \%)$ pensaient que les animaux ressentaient la douleur comme les humains. Ceci est en accord avec les résultats d'une étude menée aux Etats-Unis révélant que $60 \%$ des étudiants vétérinaires étaient d'accord sur le fait que les animaux sauvages ressentent la douleur de manière similaire aux humains (Heleski et Zanella, 2006). Une autre étude a également montré que $70 \%$ des opérateurs d'élevage en Australie étaient en désaccord avec l'affirmation selon laquelle les animaux ne ressentent pas de douleur comme les humains. De même, ces résultats sont semblables à ceux obtenus en Nouvelle-Zélande par Williams et al. (2007).

La problématique de la douleur et de la sensibilité des animaux dépend de l'espèce concernée car la sensation de douleur est liée au développement du système nerveux. Les études ont montré une hiérarchisation des conceptions sur ce sujet par les personnes enquêtées. Les investigations menées à l'Eismv montrent que $37 \%$ des étudiants, enseignants-chercheurs, et personnel administratif et technique s'accordent avec le fait que les poules, les carnivores, les porcs et les ruminants ressentent la douleur comme les humains. Cependant, 43,4\% des enquêtés pensent que ce sont les carnivores domestiques qui ressentent la douleur comme les humains. Cette hiérarchisation dans le cas des animaux domestiques place les chiens et les chats comme les animaux les plus sensibles, viennent en seconde position les porcs, puis les ruminants et enfin les volailles (Adjé Koffi, 2011). Une étude menée auprès d'étudiants vétérinaires de l'Université Cornell aux EtatsUnis montre la hiérarchisation suivante sur la question du degré de sensibilité des animaux : chien, chat, bovin, petit ruminant, porcin et volaille (Levine et al., 2005). En outre, une étude réalisée à l'Université de Cambridge au Royaume-Uni indique la hiérarchisation suivante : singe, chien, renard, porcin, volaille, rat et poisson (Phillips et McCulloch, 2005). Cette étude montre aussi une différence de perception par rapport à la nationalité : les étudiants européens sont beaucoup plus concernés par la douleur des animaux, les étudiants américains sont en deuxième position, tandis que les étudiants asiatiques sont les moins préoccupés. Pour ces trois nationalités, les personnes enquêtées pensent que les chiens et les chats ont des aptitudes cognitives plus développées que celles des autres espèces domestiques.

Le pourcentage d'étudiants favorables à l'utilisation des animaux dans la recherche scientifique $(64 \%)$ est proche de celui relevé à l'Eismv où $60 \%$ des étudiants, des enseignants-chercheurs et du personnel technique y sont favorables, avec $80 \%$ d'entre eux préférant l'utilisation d'animaux exempts de douleur, et la totalité des enseignants-chercheurs étant favorable à l'utilisation d'animaux exempts de douleur (Adjé Koffi, 2011). Mais l'enquête réalisée aux Etats-Unis chez les chercheurs et les sympathisants de la cause animale montre que seulement $43 \%$ des chercheurs sont favorables à l'utilisation d'animaux exempts de douleur (Gardner et Goldberg, 2009). Lors de cette étude, 57 \% des enquêtés montrent une grande réserve par rapport à l'utilisation des animaux dans la recherche scientifique. Ils craignent que ces animaux ne soient considérés comme des objets complexes faisant partie des équipements de laboratoire, et qu'ils soient soumis à des traitements cruels et indignes.

Certaines personnes acceptent le recours à l'expérimentation animale pour des motifs très sérieux. C'est le cas d'une enquête réalisée en France qui a montré que $56 \%$ des Français sont favorables à l'usage de l'expérimentation animale pour la découverte de vaccins ou de médicaments, et 69 à $77 \%$ le sont pour la recherche sur les maladies graves les plus connues (Verschuere et Lachapelle, 2008). L'acceptation de l'expérimentation animale pour la chirurgie est légèrement moindre $(61 \%)$. Le résultat principal de ce sondage montre que les Français acceptent très majoritairement le recours à l'expérimentation animale pour des motifs de santé sérieux.

Notre étude a montré que les étudiants de la Fastef et de la Fmpos étaient plus favorables à l'utilisation des animaux dans la recherche scientifique, ceci serait dû au fait que ces futurs instituteurs ou médecins sont plus enclins à vouloir développer le domaine de la science par le biais des expériences. 


\section{Bien-être animal et exploitation des animaux}

La grande majorité (93\%) des étudiants de l'UCAD pensait qu'il y avait une relation entre le BEA et la productivité, et la plupart d'entre eux $(89,8 \%)$ pensaient que la prise en compte du BEA dans les conditions d'élevage en Afrique permettrait d'augmenter la productivité. Ce résultat diffère de celui relevé à l'Eismv où $52 \%$ des interviewés pensent que la production animale et le BEA peuvent être complémentaires (Adjé Koffi, 2011). La grande approbation des étudiants de l'UCAD montre que leurs connaissances sur le BEA sont limitées, contrairement aux acteurs de l'Eismv qui ont des avis réservés, confirmant les modèles de McLnerney (1991), et d'Alistair et al. (2004).

Pour McLnerney, dans un élevage, il y a une corrélation entre la rentabilité de l'élevage et le BEA : lorsque le BEA est négligé, le rendement de l'élevage est compromis. Le modèle proposé par cet auteur comporte quatre points clés. Dans le premier, à l'état sauvage ou naturel, l'animal développe une «productivité naturelle », mais son bien-être n'atteint pas un niveau maximal du fait des prédateurs, des maladies, du manque de nourriture ou d'autres causes naturelles défavorables. Dans le deuxième, dans un élevage, lorsque les conditions permettant de mieux répondre aux besoins des animaux sont présentes, la production augmente et leur bien-être s'améliore. Dans un premier temps par la satisfaction de leurs besoins de base, puis par la couverture de leurs besoins secondaires, comme la protection contre les maladies ou la mise à disposition d'un abri. L'état de bien-être atteint alors son niveau maximum. Dans le troisième, les efforts menés au-delà pour améliorer la production peuvent commencer à altérer le BEA. Enfin dans le quatrième, la conduite intensive de la production atteint (ou dépasse) les limites biologiques de l'animal, altérant de manière très importante son bien-être (cela correspond au point cruauté).

McLnerney a suggéré qu'une conduite d'élevage pour une production très intensive, qui ne fournit pas les ressources permettant de répondre aux besoins individuels des animaux adaptés à leur capacité de production, pouvait entraîner une détérioration rapide du $\mathrm{BEA}$, bien au-delà de celui observé chez l'animal au stade naturel. Malheureusement pour les animaux, ils continuent d'être productifs, même quand leur bien-être est au niveau le plus bas (McLnerney, 1991).

Alistair et al. (2004) ont établi un autre modèle établissant la relation entre le BEA et la production animale. Quatre scénarios ont été décrits par cet auteur. Dans le premier, lorsque le profit est dominant et le bien-être est négligé dans un élevage, l'éleveur est gagnant et les animaux sont perdants. Dans le deuxième, lorsque le BEA est très négligé dans un élevage, le profit est minimum, et l'éleveur et les animaux sont tous perdants. Dans le troisième, l'état idéal pour une ferme est de mettre au même niveau le profit et le BEA, créant ainsi une harmonie parfaite. L'élevage devient rentable quand les attentes respectives de l'animal et de l'éleveur sont atteintes simultanément. Enfin dans le quatrième, lorsque dans un élevage le BEA est très dominant et le profit est minimum, l'éleveur est perdant mais les animaux sont gagnants.

Les modèles de ces deux auteurs aboutissent à la même conclusion : il faut évaluer correctement les compromis entre le profit et le BEA au niveau même de l'exploitation. Pour ce faire, il suffit d'identifier et d'estimer les coûts cachés et les avantages des décisions au niveau des exploitations. Ensuite, il faut avoir une très bonne connaissance de l'état physique des animaux, c'est-à-dire de leur bien-être, et une parfaite intégration de cette notion dans la modélisation économique au niveau des fermes d'élevage est nécessaire.

\section{CONCLUSION}

Plus de la moitié $(57 \%)$ des étudiants de l'UCAD ont entendu parler au moins une fois du BEA, 60,4\% parmi eux à travers les médias. Les étudiants de première et deuxième années étaient plus en accord avec le fait que le BEA est une question primordiale en Afrique, ce qui laisse espérer une intégration future du BEA dans les élevages en Afrique. Néanmoins, une sensibilisation pour améliorer les connaissances sur ce concept est nécessaire et devra se faire à travers les médias qui constituent le canal le plus utilisé par les étudiants de l'UCAD.

\section{REFERENCES}

Adjé Koffi J.F., 2011. Perception du bien-être animal par les acteurs de la communauté de L'Eismv de Dakar. Thèse Doct., Ecole inter-Etats des sciences et médecine vétérinaires de Dakar, Dakar, Sénégal ( $\left.n^{\circ} 8\right)$

Altinok N., 2005. L'apport des enquêtes internationales à la mesure qualitative du capital humain. Iredu, Dijon, France, $23 \mathrm{p}$.

Burgat F., 2001. La demande concernant le bien-être animal. Courr. Environ. INRA, 44: 65-68

Coleman G., 2009. La perception sociale de la douleur animale. Rev. Sci. Tech. Off. Int. Epizoot. (10) : 26-37

Coleman G.J., Hay M., Toukhsati, 2005. Effects of consumer attitudes and behaviour on the egg and pork industries. Report to Australian Pork Ltd and Australian Egg Corp. Monash University, Clayton, Australia

Farm Animal Welfare Council, 1992. FAWC updates the five freedoms. Vet. Rec. (17): 357

Gardner R.M., Goldberg A.M., 2009. La production d'animaux " exempts de sensibilité à la douleur " est-elle une solution de remplacement acceptable? Rev. Sci. Tech. Off. Int. Epizoot. (10) : 57-78

Heleski C.R., Zanella A.J., 2006 Animal sciences student attitudes to farm animal welfare. Anthrozoos, 19 (1): 3-16

Lawrence A.B., Conington J., Simm G., 2004. Breeding and animal welfare: practical and theoretical advantages of multi-trait selection. Anim. Welfare, 13: S191-S196

Levine F.D., Mills D.S., Houpt K.A., 2005. Attitudes of veterinary students at one US college towards factor relating to animal welfare. J. Vet. Med. Educ., 32 (4): 481-490

McLnerney J.P., 1991. Economic aspects of the animal welfare issue. In: Proc. Society of Veterinary Epidemiology and Preventive Medicine, London, UK, p. 83-91

OIE, 2010. Code sanitaire pour les animaux terrestres. Introduction sur les recommandations relatives au bien-être animal. OIE, Paris, France, articles 7.1 .1 à 7.1 .3

OIE, 2012. Bien-être animal. www.oie.int/fr/bien-etre-animal/themesprincipaux/ (consulté le 13 avril 2012)

Phillips C.J.C., McCulloch S., 2005. Attitudes of students of different nationalities toward animal sentience and the use of animal in society, with implication for animal use in education. J. Biol. Educ., 40 (1): 17-24

Université Cheikh Anta Diop, Dakar, Sénégal. www.ucad.sn/index. php?option $=$ com_content $\&$ view $=$ article $\& i d=252 \&$ Itemid $=277$ ) (consulté le 10 janvier 2012)

Verschuere B., Lachapelle F., 2008. Résultats d'un sondage relatif à I'expérimentation animale en France. Bull. Acad. Vét. Fr., 161 (5) : 393-397

Williams V.M., Dacre I.T., Elliot M., 2007. Public attitudes in New Zealand towards the use of animals for research, testing and teaching purposes. N. Z. J., 55 (2): 61-68 


\section{Summary}

Dutuze M.F., Issa Garba A.R., Manishimwe R., Kone P. Perception of animal welfare by Cheikh Anta Diop University's students

Animal welfare is a very complex concept to define, but it is important for the quality of livestock products. To assess the perception of this concept, a study was conducted with students from the University of Cheikh Anta Diop (UCAD) in Dakar between January 16 and May 2, 2012. The student population was 84,396 at time of study. In total 168 students were interviewed using a structured questionnaire. The results showed that only $57 \%$ of them had heard of animal welfare at least once and $60.4 \%$ among them via media. Students' knowledge on animal welfare varied between schools: students of the School of Sciences and Technology of Education and Training (FASTEF) were the most informed. Moreover, $73.2 \%$ believed that animals are sentient and $93 \%$ that there is a relationship between animal welfare and productivity. It is essential to make students aware of this concept and to do so via media, because media are the channels of information the most used by UCAD students.

Keywords: animal welfare, pain, awareness raising, student, productivity, Senegal

\section{Resumen}

Dutuze M.F., Issa Garba A.R., Manishimwe R., Kone P. La percepción de bienestar de los animales por parte de los estudiantes de la Universidad Cheikh Anta Diop de Dakar

El Bienestar de los Animales (BEA) es un concepto muy complejo de definir, pero es importante para la calidad de los productos ganaderos. Para evaluar la percepción de este concepto, se realizó un estudio dentro de la comunidad estudiantil de la Universidad Cheikh Anta Diop de Dakar (UCAD), entre el 16 de enero y el 2 de mayo de 2012. Durante este periodo la población estudiantil era de 84 396. Se entrevistó una muestra de 168 estudiantes, mediante un cuestionario estructurado. El estudio mostró que sólo el $57 \%$ de los encuestados había escuchado al menos una vez hablar de BEA, del cual $60,4 \%$ a través de los medios de comunicación. Los conocimientos de los estudiantes sobre la BEA variaron de una escuela a otra: los estudiantes de la Facultad de Ciencias y Tecnologías de la Educación y Formación (FASTEF) estaban mejor informados. Entre los encuestados, $73,2 \%$ cree que los animales sienten dolor y $93 \%$ de que existe una relación entre BEA y la productividad. Es necesaria una sensibilización de este concepto y debe hacerse a través de los medios de comunicación, que son el canal más utilizado por los estudiantes de UCAD.

Palabras clave: Bienestar animal, dolor, sensibilización, estudiante, productividad, Senegal 


\section{Matériel supplémentaire}

\section{Questionnaire}

Opinions des étudiant(e)s de l'université Cheikh Anta Diop de Dakar sur le bien-être animal

Fiche $n^{\circ}$ :

Date : (JJ/MMIAAAA)

\section{IDENTITE}

1. Sexe : $\mathrm{M} \square \mathrm{F} \square$

2. Age (ans): $15-20$

21-26

27-32

$>32$

3. Faculté ou école :

4. Niveau d'étude : $1 \mathrm{~A}$

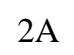

$3 \mathrm{~A}$

$4 \mathrm{~A}$

$5 \mathrm{~A}$

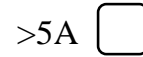

\section{RELATION A VEC LES ANIMAUX}

5. Avez-vous un animal ? Oui $\square$ Non

6. Si oui lequel ? Animal de compagnie

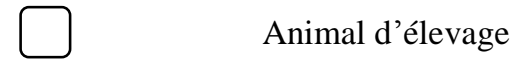

7. Aimez-vous les animaux ? Pas du tout

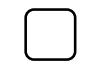

Un peu

Plus ou moins

Beaucoup

Ne sais pas

\section{CONNAISSANCES GENERALES SUR LE BIEN-ETRE ANIMAL}

8. Avez- vous entendu parler une fois de la notion de « bien-être animal » ?

Oui Non

9. Si oui par quel canal d'information ?

En cours

Par les médias Conférences

10. Comment deffinissez-vous le bien être animal ?

\section{L'absence de faim et de soif$$
\text { Le confort physique }
$$

La bonne santé et l'absence de blessure et/ou de douleurs

La possibilité d'exprimer le comportement normal de l'espèce

L'absence de peur et de détresse

Autres

11. Avez-vous déjà entendu parler d'associations de lutte pour le bien-être animal ?

Pas du tout

Un peu

Beaucoup 


\section{OPINIONS SUR LE RESPECT DU BIEN-ETRE ANIMAL DANS NOS} CONDITIONS D'ELEVAGE

$\begin{array}{lllll}1 & 2 & 3 & 4 & 5\end{array}$

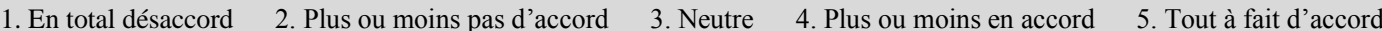

12. Selon vous le bien-être est-il une question primordiale?

13. Pensez-vous que le bien-être animal est respecté dans nos conditions d'élevage (Afrique) ?

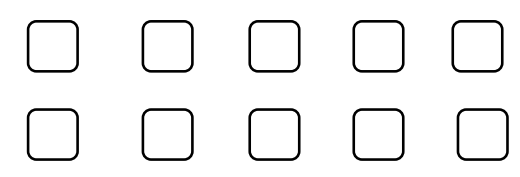

V.SENSIBILITE DES ANIMAUX ET RECHERCHE SCIENTIFIQUE

14. Pensez-vous que les animaux ressentent la douleur ?

1. Moins que l'homme

$1 \square$

2. Comme l'homme

3. Plus que l'homme

4. Ne sais pas

4

15. Êtes-vous favorable à l'utilisation des animaux dans la recherche scientifique ?
1. Très défavorable
2. Moyennement défavorable
3. Un peu défavorable
6. Moyennement favorabl
5. Un peu favorable

7. Très favorable

4. Neutre

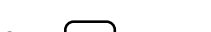

1

2

4

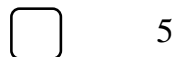

5

6

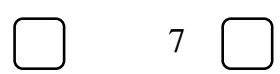

16. Expliquez votre réponse

\section{BIEN-ETRE ET EXPLOITATION DES ANIMAUX}

17. D'après vous, y a-t-il une relation entre le bien-être animal et la productivité animale ? Oui $\square$ Non $\square$

18. Si oui pensez-vous que la prise en compte du bien-être de l'animal dans nos élevages permettrait d'augmenter la productivité ?
Pas du tout
Un peu
Beaucoup
Ne sais pas

19. Etes-vous favorable à l'utilisation des animaux en exploitation agricole ?
1. Très défavorable
2. Moyennement défavorable
3. Un peu défavorable
5. Un peu favorable
6. Moyennement favorable
7. Très favorable
4. Neutre<smiles>[AlH]</smiles>
2

3

4
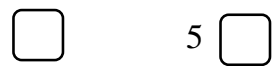

6

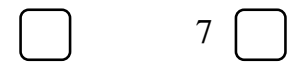

20. Pensez-vous que les animaux sont maltraités en exploitation agricole ?

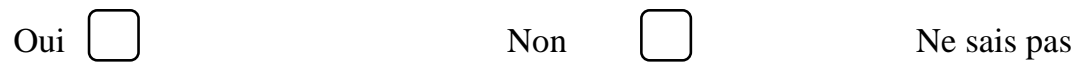

21. Selon vous l'utilisation de certains animaux (comme le cheval) pour le transport est-elle une violation du concept de bien-être animal ?

Oui

Non

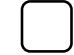

$\mathrm{Ne}$ sais pas 